\title{
Evaluation of the Chemical Integrity of Beta-Lactam Antibiotics by Iodine-Based Assay
}

\author{
Abraham Salois', Iris Perez², Ervin Palma3 , Ethan Goolish4, Yuri Griko5* \\ ${ }^{1}$ Chief Dull Knife College, Lame Deer, USA \\ ${ }^{2}$ Evergreen Valley College, San Jose, USA \\ ${ }^{3}$ California State University Eastbay, Hayward, USA \\ ${ }^{4}$ Mountain View High School, Mountain View, USA \\ ${ }^{5}$ National Aeronautics and Space Administration, Ames Research Center, Moffett Field, USA \\ Email: "Yuri.V.Griko@nasa.gov
}

Received 3 October 2015; accepted 20 November 2015; published 23 November 2015

Copyright (C) 2015 by authors and Scientific Research Publishing Inc.

This work is licensed under the Creative Commons Attribution International License (CC BY). http://creativecommons.org/licenses/by/4.0/

c) (i) Open Access

\begin{abstract}
One of the major problems associated with the therapeutic application of $\beta$-lactam antibiotics is their significant instability [1]-[3]. Analogs from that group are easily degraded in aqueous solutions and in solid state, which happens predominantly as a result of $\beta$-lactam ring chemical degradation. Because of this, the chemical integrity of $\beta$-lactam antibiotics must be closely monitored to meet FDA requirements. The original iodometric method based on visual detection of the end products of beta-lactamase hydrolysis that has been used to test beta-lactamase activity [4] was modified to quantitatively assess the amount of degraded product in amoxicillin. A UV-visible spectrophotometric method has been used, focusing on the ability of the products from the degradation of $\beta$-lactam analogs to absorb iodine from bulk solution, resulting in a significant reduction in the absorption spectra. Amoxicillin trihydrate tablets with different expiration dates and amoxicillin samples pre-loaded with known amounts of degraded amoxicillin have been used to generate a calibration curve and quantify the presence of degraded materials. Results show that the changes in the absorption spectra of iodine reagent directly correlate with the fraction of degraded $\beta$-lactam antibiotics, such as amoxicillin, in commercial products. The proposed assay might be as close as one step from simpler and cheaper methods of quantitative analysis of degraded $\beta$-lactam analogs, with estimated sensitivity in the range of $1 \%-2 \%$, which is better than the current FDA requirements.
\end{abstract}

${ }^{*}$ Corresponding author.

How to cite this paper: Salois, A., Perez, I., Palma, E., Goolish, E. and Griko, Y. (2015) Evaluation of the Chemical Integrity of Beta-Lactam Antibiotics by lodine-Based Assay. Journal of Biosciences and Medicines, 3, 91-99.

http://dx.doi.org/10.4236/jbm.2015.311012 


\section{Keywords}

\section{Amoxicillin, Iodine, Chemical Integrity}

\section{Introduction}

$\beta$-lactam antibiotics have a broad spectrum of antibacterial uses and have such been successfully used in the treatment of a large number of diseases with bacterial etiology. The common element in the chemical structure of all $\beta$-lactam antibiotics is the presence of a five-membered $\beta$-lactam ring. Due to this underlying chemical specificity, they are easily degraded in both aqueous solution and in solid state [1]-[3]. This chemical degradation may result not only in the loss of antibacterial efficiency, but also in the appearance of strong allergy-provoking properties as a result of their degradation products. The amount and type of degradation products of $\beta$-lactam antibiotics often depend on a few different affecting factors (solvents, concentration of substance and hydrogen ions, temperature, etc.), causing concern for the stability of $\beta$-lactam antibiotics in solution. A reliable, simple, and quantitative analytical method for the determination of $\beta$-lactam integrity is therefore necessary to control the degree of degradation of these antibiotics. Most, if not all, analytical methods for integrity analysis of $\beta$-lactam analogs were developed based on the surveying of spectrophotometric properties in a visible and ultraviolet radiation range [5]-[12]. The desired signals were possible to obtain by measurement of either direct absorption or of the absorption of the resulting species of an analyte/derivatizing reagent reaction [5]-[13].

One of the fastest and simplest methods for routine measurements of the integrity of the $\beta$-lactam ring of this group of antibiotics is based on the reduction of iodine by the hydrolyzed substrate [4] [12]. Various modifications of the original method have been introduced for specific assays required for different $\beta$-lactam antibiotics [4], being predominantly focused on a quantitative estimate of $\beta$-lactamase activity [4]. In general, quality control for the chemical integrity of this kind of antibiotics has been assigned to more sophisticated methods, such as mass spectroscopy, liquid chromatography, and vibrational spectroscopy (RAMAN, FTRI) [5] [13] [14]. However, in spite of the high accuracy and high resolution of these methods, they remain expensive, require professional training, and need special samples to be prepared for such fixed-time assay. Current guidelines from regulatory agencies such as the International Conference on Harmonization (ICH) and the Food and Drug Administration (FDA) have introduced certain requirements and restrictions for the development and application of analytical methods for the analysis of $\beta$-lactam antibiotics and their related products [15] [16]. Even using many of these analytical techniques, it is still challenging to quantitatively determine if the product meets the $10 \%$ degradation or less benchmark required by the FDA to stay on the market [16]. This is why the search for new analytical methods, especially convenient, time-fixed, and inexpensive ones, is very important. Specifically, spectrophotometric properties of $\beta$-lactam analogs (with accuracy and selectivity enhanced by chemometric procedures) have been gaining significant interest [5]-[12]. In this report, we describe a modification of the original iodometric method [4] that allows for the detection of degradation in $\beta$-lactam antibiotic products with integrity of less than $90 \%$. Amoxicillin before as well as after the expiration date from different manufacturers has been used in this study as examples.

\section{Materials \& Methods}

In order to produce a quantitative positive control for $\beta$-lactam ring integrity, amoxicillin was hydrolyzed by alkaline hydrolysis [4]. The concentration and amount of $\beta$-lactam degraded analyte in the presence of iodine reagent was calculated from the following information (All reagents used in this study were of analytical grades):

Stock iodine solution was prepared by adding $0.7 \mathrm{~g}_{\text {of } \mathrm{I}_{2}}$ with $3.45 \mathrm{~g}$ of $\mathrm{KI}$ and dissolving it in $17.24 \mathrm{~mL}$ of distilled water.

Iodine reagent was prepared as described previously for the original fixed-time assay method [2]. Briefly, $5 \mathrm{~mL}$ of stock iodine solution was added to $95 \mathrm{~mL}$ of the acetate buffer ( $80 \mathrm{~g}$ of anhydrous sodium acetate adjusted to $\mathrm{pH}$ 4.0 with acetic acid and brought to 2 liters with distilled water). The stock iodine solution contained $0.16 \mathrm{M}$ iodine and $1.2 \mathrm{M}$ potassium iodide (20.3 $\mathrm{g}$ of iodine and $100 \mathrm{~g}$ of potassium iodide dissolved in $500 \mathrm{~mL}$ of distilled water).

A Lambda 35 UV-double beam spectrophotometer (Perkin Elmer Inc.) with a $1 \mathrm{~mm}$ length quartz cuvette was used for absorbance measurements. 
Amoxicillin samples were prepared by the solubilization of amoxicillin powder (500 mg capsule) in $20 \mathrm{mM}$ Na-P buffer, $\mathrm{pH} 7.0$ at a final concentration of $2.5 \mathrm{mg} / \mathrm{mL}$. Both a commercially available TEVA brand of amoxicillin and a commercially available Westward brand of amoxicillin were used for this study.

Degraded amoxicillin was prepared as follows, reproduced as according to [4]. A $2 \mathrm{~mL}$ solution of $50 \mathrm{mg}$ of amoxicillin in $0.2 \mathrm{M} \mathrm{NaOH}$ was incubated at $30^{\circ} \mathrm{C}$ for 30 minutes, at which point the solution was neutralized in an ice bath with an equal amount of $0.2 \mathrm{M} \mathrm{HCl}$. $16 \mathrm{~mL}$ of $20 \mathrm{mM}$ Na-P buffer, $\mathrm{pH} 7.0$ was used to bring the degraded amoxicillin to a concentration of $2.5 \mathrm{mg} / \mathrm{mL}$.

To evaluate the degradation of amoxicillin in suspension over time, several identical suspensions were prepared and stored at room temperature $\left(23^{\circ} \mathrm{C}\right)$ in closed containers, each for a selected period of time. These samples were later assayed by iodometric titration using a spectrophotometric method.

\section{Results and Discussion}

Figure 1 shows a schematic for the changes in the chemical structure of the $\beta$-lactam ring of amoxicillin as it degrades, starting with its completely intact form (top), followed by the structure once the ring has been broken (middle), and finally with the consumption of the iodine molecules from the bulk iodine solution (bottom), leading to a decrease in the absorbance spectra of iodine at $350 \mathrm{~nm}$ as shown in Figure 2.

Figure 2 shows the intrinsic spectra of amoxicillin (a) and iodine reagent (b) in a UV-visible wavelength range. The spectra of amoxicillin have a maximum at $280 \mathrm{~nm}$ while iodine reagent has two maximums in its spectra, at $290 \mathrm{~nm}$ and $350 \mathrm{~nm}$. The absence of absorbance in amoxicillin's spectra at $350 \mathrm{~nm}$ makes it convenient to monitor the presence of free iodine at this wavelength in an amoxicillin/iodine mixture. Adding amoxicillin to the iodine

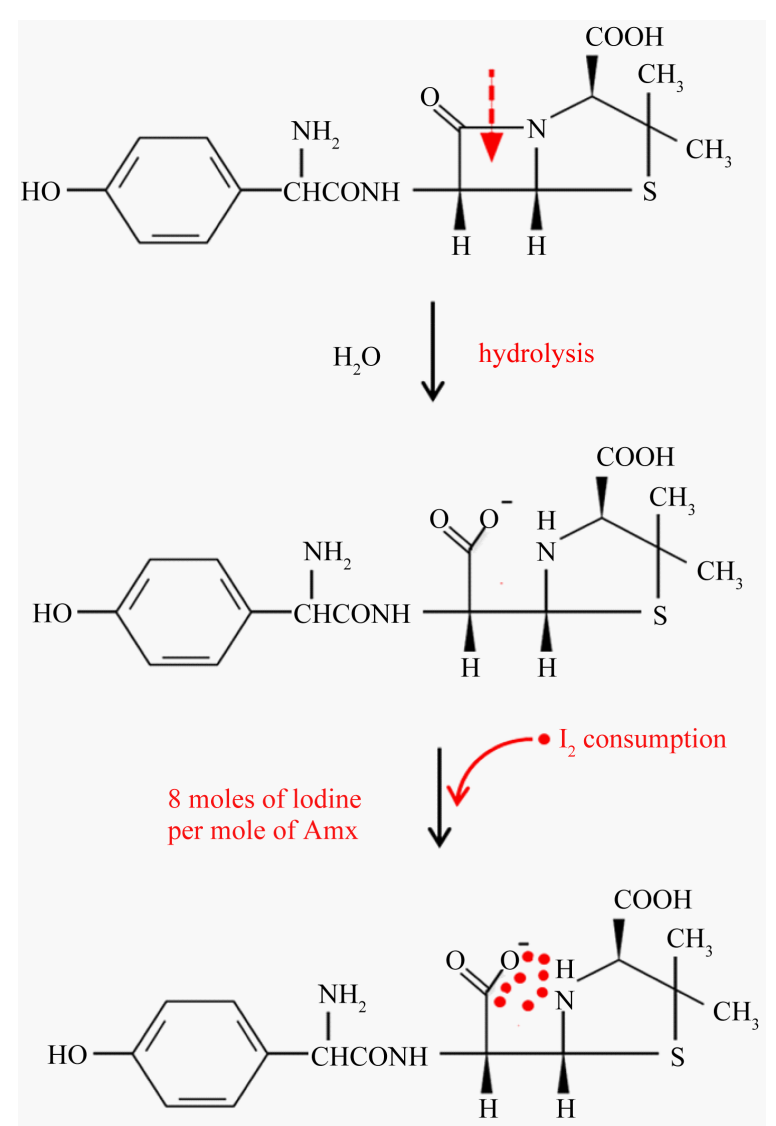

Figure 1. Schematic presentation of changes in the chemical structure of the $\beta$-lactam ring of amoxicillin starting with its completely intact form (top) followed by the structure once the ring has been broken (middle) and finally with the consumption of iodine molecules in the bulk iodine solution (bottom). 
solution resulted in a decrease of the absorbance at $350 \mathrm{~nm}$ due to the consumption of iodine by degraded amoxicillin from a bulk solution (Figure 2(d)). Since iodine binds only to open lactam ring (degraded) amoxicillin, it is absorbed by the degraded molecules in hydrolyzed amoxicillin (Figure 2(c)), but only until the degraded material is saturated.

It is interesting to note that amoxicillin degraded after a 20 min incubation period in $0.2 \mathrm{~N} \mathrm{NaOH}$ solution, leading to the complete hydrolysis of the antibiotic [4], shows no visible changes in the absorbance spectra in the entire range between 240 - $600 \mathrm{~nm}$, even though the $\beta$-lactam ring underwent degradation. This indicates that the $\beta$-lactam ring opening itself does not produce absorbance signals in the visible region of radiation [7].

Figure 3 presents changes in the UV-spectra of an amoxicillin/iodine solution and also its absorbance at 350 $\mathrm{nm}$ during the titration of amoxicillin with an iodine reagent. Adding the iodine reagent to the solution of intact amoxicillin (Figure 3(b)) produces a linear titration curve, similar to that of in calibration blank test, where iodine reagent was added to the buffer solution without amoxicillin (Figure 3(a)).

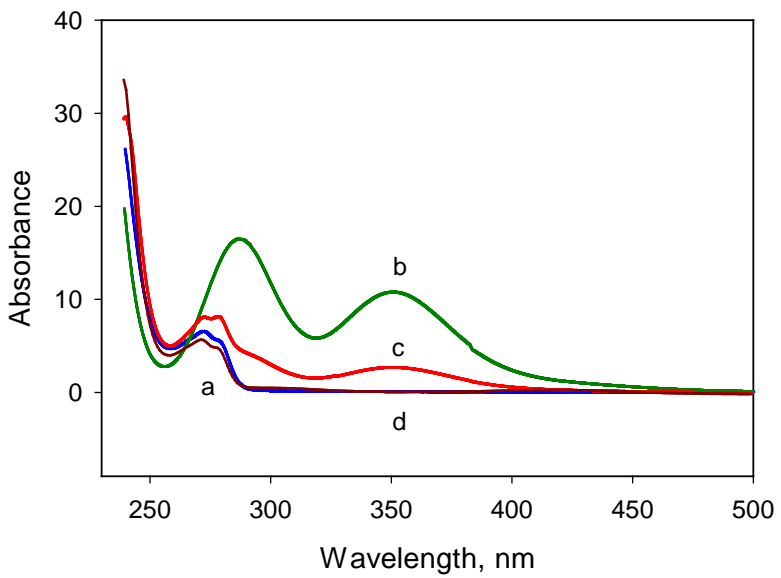

Figure 2. UV-visible spectrums of amoxicillin (a) and the iodine reagent (b) in buffer solution (20 mM Na-P, $\mathrm{pH} 7.0$ ); solution of partially degraded amoxicillin with the iodine reagent added (b); and a completely degraded amoxicillin sample (d).

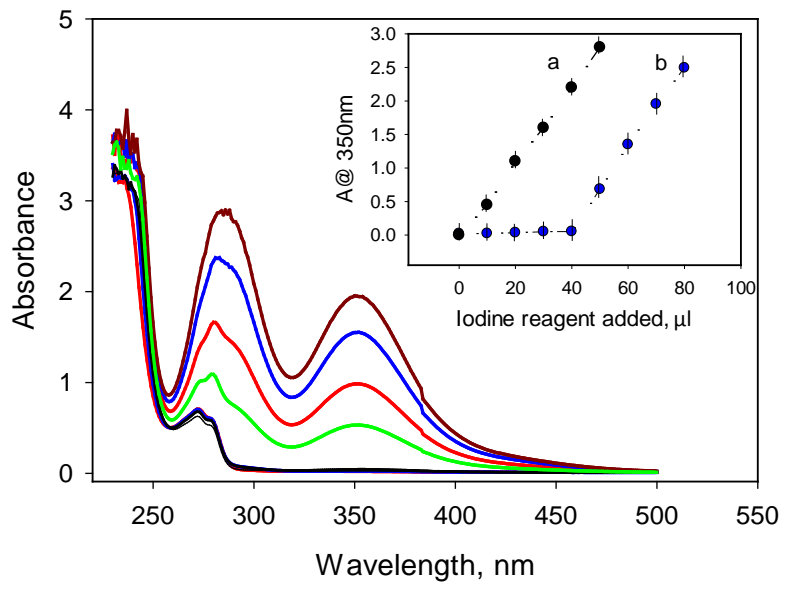

Figure 3. Changes in the UV-spectra of amoxicillin/iodine solution with an increased concentration of iodine reagent. Each color represents UVspectra for individual titration point. The insert shows a titration plot of the iodine reagent added to a blank buffer solution without amoxicillin (a) and to amoxicillin (b) at ambient temperature, as measured by changes in UVabsorbance at $350 \mathrm{~nm}$. The concentration of amoxicillin in the starting solution of $20 \mathrm{mM}$ Na-P buffer, $\mathrm{pH} 7.0$, was $2.5 \mathrm{mg} / \mathrm{mL}$. The concentration of iodine in stock solution of iodine reagent was $0.16 \mathrm{M}$. 
However, the presence of degraded amoxicillin in a sample resulted in the reduction of iodine as described previously, leading to practically no changes in the absorbance at the spectrum peak at $350 \mathrm{~nm}$, as the iodine added was consumed by the hydrolyzed amoxicillin. After the saturation point, the changes in absorbance increase linearly in a fashion similar to that of free iodine in solution. As follows from the plot, these two linear regions of the concentration-dependent function meet at a saturation point for the given concentration of amoxicillin in solution. For the sample presented, this point is reached after adding $40 \mu \mathrm{L}$ of iodine reagent to the amoxicillin (concentration of $2.5 \mathrm{mg} / \mathrm{mL}$ ).

Figure 4 shows the results for titrations of amoxicillin solution samples containing different fractions of degraded molecules. The standard hydrolyzed amoxicillin solution was prepared as described in M\&M. In each sample, a precise fraction of degraded amoxicillin was added to an intact solution of the same concentration in the range of "contamination" from 0 to $100 \%$.

Each data point was taken 3 times and then averaged to determine a statistically sound interval. The slope of each sample increases very slowly along the $\mathrm{x}$-axis, as iodine reagent is added, until the saturation point is reached. From that point on, the added iodine acts like it does in free solution, contributing greatly to the slope of the sample. As is evident from the figure, the saturation point (the point at which there is no more degraded amoxicillin to react with the iodine reagent) correlates directly with the percentage of degraded material; given any future sample, a titration until the point of saturation would give us a clear measure of the substance's integrity.

The stoichiometry of iodine molecules binding to molecules of degraded amoxicillin was determined from a titration of a $100 \%$ degraded amoxicillin solution with iodine reagent of a known concentration. Given an amoxicillin concentration of $2.5 \mathrm{mg} / \mathrm{mL}$ and a saturation point of 355 microliters of reagent, the molar ratio was calculated to be 1:6. This stoichiometry coefficient could then be used for quantitative analysis of amoxicillin samples with unknown fractions of degraded amoxicillin.

Using this knowledge, the calculations show that the concentration of degraded amoxicillin in our solution was $2.3 \mathrm{mg} / \mathrm{mL}$. This was very close to the real concentration of the initially prepared amoxicillin solution, measured spectrophotometrically using the molar extinction coefficients $\varepsilon_{274} \mathrm{mM}=1.4$ and $\varepsilon_{230} \mathrm{mM}=10.85$. The small difference between these values may be associated with the kinetic factor of iodine discoloration.

In order to demonstrate the accuracy of the method, the titration was performed again with a smaller fraction of added iodine ( $5 \mu \mathrm{L}$, not shown). Based on the consistent distance between each slanted line, each representing an additional $5 \%$ of degraded material, we can interpolate based on the location of the fresh amoxicillin sample to determine that its integrity is mostly intact, with only $2 \%-3 \%$ of degraded material. This in itself strikes an interesting observation; even amoxicillin that has not reached its expiration date contains some percent of degradation.

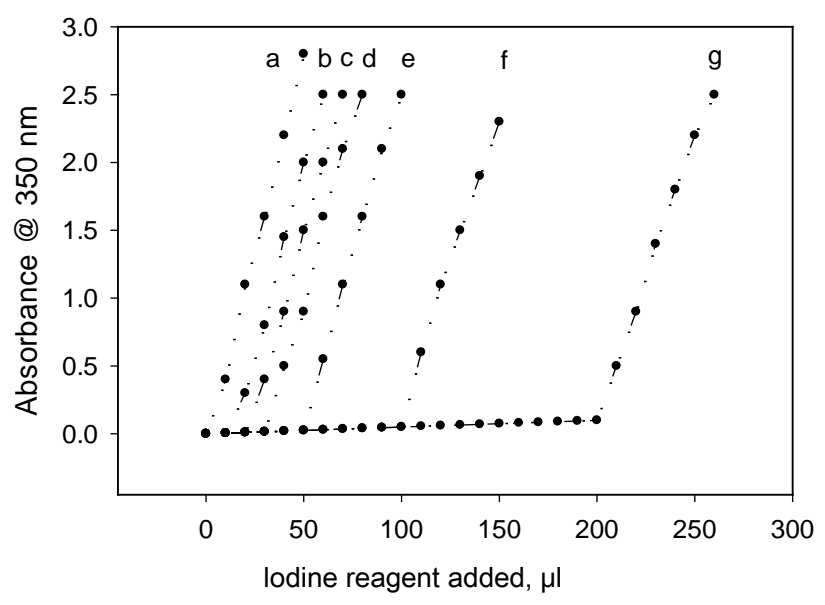

Figure 4. The iodine titration plots of an amoxicillin solutions containing different fractions of degraded molecules, which were prepared by adding certain amounts of the hydrolyzed amoxicillin to freshly prepared amoxicillin of the same concentration. The percentage of the degraded amoxicillin in the tested sample is: (a) blank solution; (b) $3 \%$; (c) $6 \%$; (d) $9 \%$; (e) $14 \%$; (f) $28 \%$; (g) $56 \%$. 
Since amoxicillin tends to degrade by a wide variety of chemical reactions, such as oxidation, photo-degradation, and racemization, the effect of these reactions may interfere with the iodine titration assay. The yellowish color development of amoxicillin is generally considered as a visual criterion of the presence of degradation products. Therefore, we have tested how the development of a yellowish color of amoxicillin solution may interfere with the quantitative estimation of the $\beta$-lactam ring degradative material in a sample.

Figure 5 shows the changes over time for the absorbance of an amoxicillin solution at $350 \mathrm{~nm}$ during storage at ambient temperature in solution with a $\mathrm{pH}$ of 2.0 and a $\mathrm{pH}$ of 7.0. The absorption at $350 \mathrm{~nm}$, which was not detectable in the intact amoxicillin, increases as the amoxicillin in solution degrades. Its degradation closely correlated with a yellow color development over the same time period, as evident from a visual monitoring of the samples.

Figure 6 shows that the recorded spectra of amoxicillin solution undergo a significant transformation in shape as either degradation proceeds over time (Figure 6(c)) or as induced by a short time exposure to high temperature (Figure 6(b)), which indicates an additional contribution to the absorbance at $350 \mathrm{~nm}$ due to the color development.

Figure 7 portrays an unaltered chart displaying the absorbances of various amoxicillin samples in solution as iodine reagent is added. In this case, the stock solution of the hydrolyzed amoxicillin used for the inoculation of

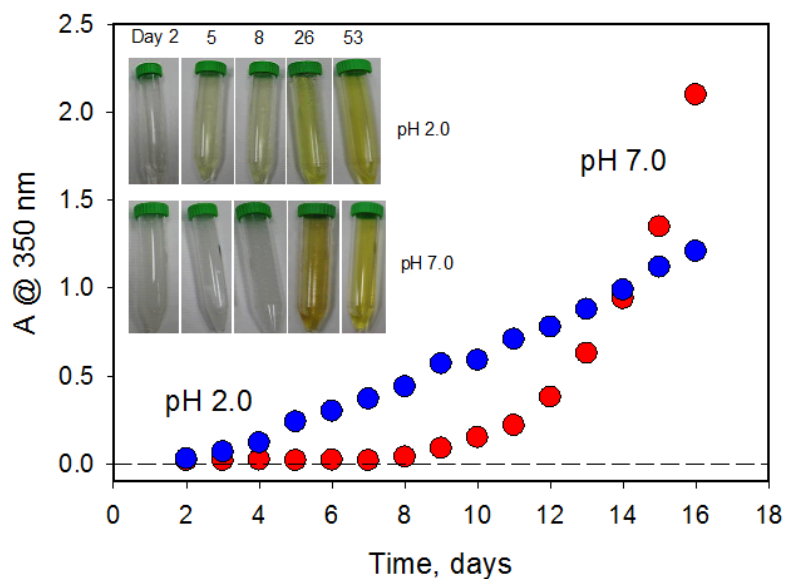

Figure 5. Kinetics of amoxicillin degradation in solution with $\mathrm{pH} 7.0$ and $\mathrm{pH} 2.0$ as measured by UV absorbance at $350 \mathrm{~nm}$ and yellowish color development.

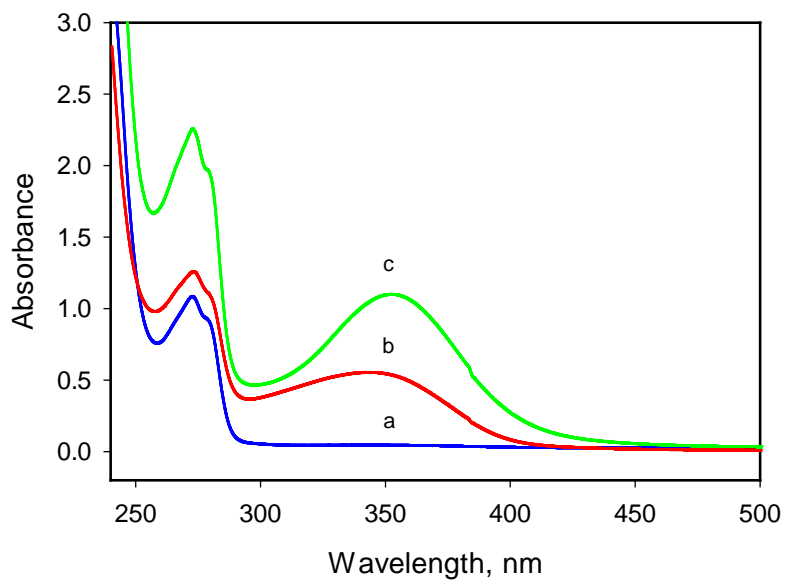

Figure 6. UV-spectra of intact and degraded amoxicillin: (a) is the intact amoxicillin in a solution of $20 \mathrm{mM}$ Na-P, pH 7.0; (b) represents heat degraded amoxicillin after a heating to $130^{\circ} \mathrm{C}$ in the same buffer solution; (c) represents amoxicillin in $20 \mathrm{mM}$ Na-P buffer, $\mathrm{pH} 7.0$ after 14 days storage at $25^{\circ} \mathrm{C}$. 


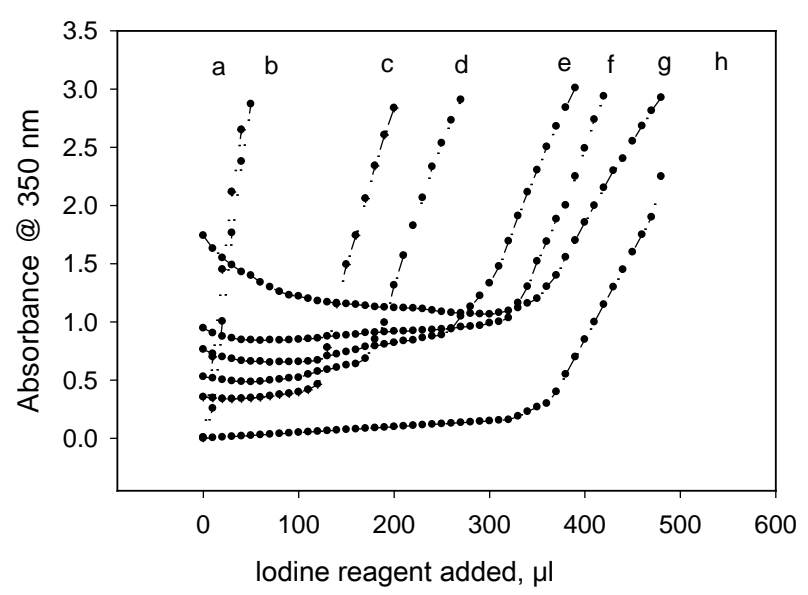

Figure 7. The iodine titration plots of an amoxicillin solutions containing different fractions of degraded molecules. The unedited changes in the absorbance of amoxicillin samples observed before the saturation point are associated with a developing yellowish color of the hydrolyzed stock of amoxicillin developed over time of storage. The percentage of the degraded amoxicillin in the tested sample is: (a) blank solution; (b) Fresh; (c) 20\%; (d) $40 \%$; (e) $60 \%$; (f) $80 \%$; (g) 1 week old $100 \%$ degraded; (h) Newly prepared $100 \%$ degraded.

resized amount of the degraded amoxicillin in each sample was not freshly prepared, but instead stored over one week for the development of yellow color. As is the case with the other figures given, the saturation points seem to directly correlate with the percentage of degraded material. What is unique about this figure in particular are the rising starting absorbances, which then slope downward as iodine is added until the saturation point. The explanation for this phenomenon is that it is due to the discoloration of amoxicillin mentioned previously. However, this discoloration does not affect the integrity analysis method described. As iodine reagent is added, it reacts with the discolored degradation products and returns the absorbance to a semi-regular number, similar to as if the solution had not degraded over time at all; in essence, the saturation point is unchanged.

Figure 8 shows the iodine titration curves of amoxicillin produced after 2 and 9 days of storage in a $\mathrm{pH} 7.0$ solution at ambient temperature $\left(23^{\circ} \mathrm{C}\right)$, when no yellow color had been developed yet (Figure 5). As shown in both cases, the saturation points differ in direct correlation with the solutions' time of storage, indicating that the amount of amoxicillin degraded through the opening of the $\beta$-lactam ring (to which iodine specifically binds) progressively increases over time. The amoxicillin at ambient temperature on the second day of storage had about $8 \%$ impurity increasing to $23 \%$ on the ninth day.

Together with the results presented in Figure 4, this demonstrates that the proposed method allows for the quantitative measurement of the amount of degraded material in amoxicillin, something that is not truly evident by the visible colorization of amoxicillin solution.

Another find in this study suggests that the rate of degradation of amoxicillin in solution is substantially faster than determined in earlier studies. An increase by $5 \%$ in degraded products happens in the first day of storage at $23^{\circ} \mathrm{C}$ vs. the 7 days found in previous studies [17]. While the degradation of amoxicillin through $\beta$-lactam ring hydrolysis was progressing over time of storage, the stored samples did not show any sign of yellow color over the first several days. This indicates that beta-lactam ring degradation is not associated with color development, which may occur through another pathway(s) of degradation.

In order to estimate the effect of ionic strength on iodine titration results, comparative measurements were performed in absence of and in presence of $0.1 \mathrm{M}$ sodium chloride. Results show that for freshly prepared amoxicillin solutions, the iodine titration curves are essentially the same for both the solution containing $\mathrm{NaCl}$ and the solution without. This clearly indicates that the interaction of iodine reagent with amoxicillin during titrations is unaffected by the presence of such a salt. Therefore, variations of ionic strength for a range of concentrations of $\mathrm{NaCl}$ do not affect the chemical reaction between iodine and the $\beta$-lactam ring of amoxicillin. In addition, the presence of $\mathrm{NaCl}$ in amoxicillin solution was found not to affect the rate of degradation for amoxicillin during storage at room temperature (data not shown). 
The experimental results presented in this study demonstrate a potential for a practical application of the iodometric method, allowing for a fast and inexpensive evaluation of $\beta$-lactam antibiotics for impurities caused by $\beta$-lactam ring degradation. Figure 9 compares the saturation points of four different freshly prepared amoxicillin samples with different expiration dates as well as the saturation point of a blank sample containing only the buffer solution.

This shows that freshly prepared samples made with amoxicillin past its expiration date contain more degraded material than do the freshly prepared samples from new amoxicillin. When tested in this study, the iodometric assay allows for the detection of $\beta$-lactam degraded material in amoxicillin commercial products, with an accuracy of $\sim 2 \%$. Our study also has indicated that freshly prepared amoxicillin suspensions made from $500 \mathrm{mg}$ tablets of different manufacturers and taken prior to the expiration date may contain up to $3 \%$ of degraded amoxicillin.

In addition, development of a product assay using starch iodine as a color indicator could be based on the research done above. By comparing the intensity of a colored reaction (between $\beta$-lactam antibiotics and the starch iodine) to that of a quantitative standard, the percent of the degraded products in a stored container could be determined, similar to the technique previously developed based on an iodine-starch paper strip [14].

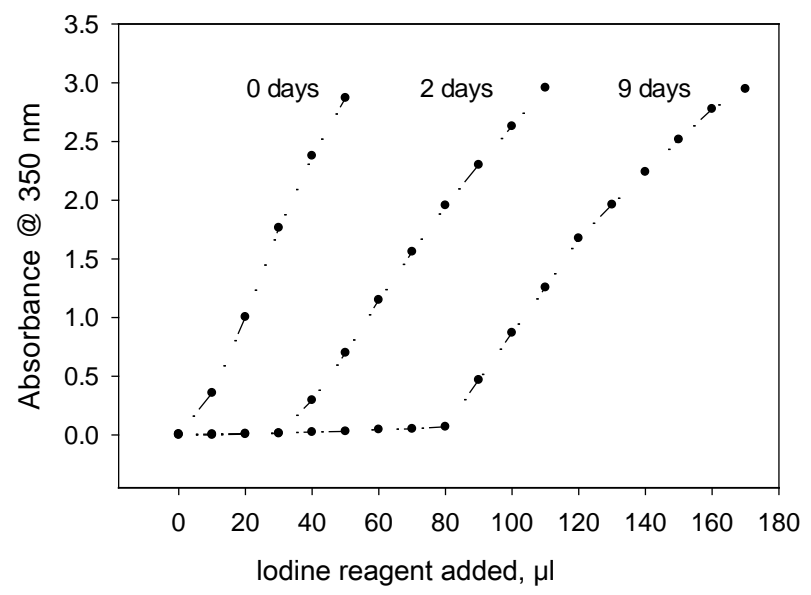

Figure 8. The iodine titration curves of amoxicillin produced after 2 and 9 days of storage in a $\mathrm{pH} 7.0$ solution at ambient temperature $\left(23^{\circ} \mathrm{C}\right)$.

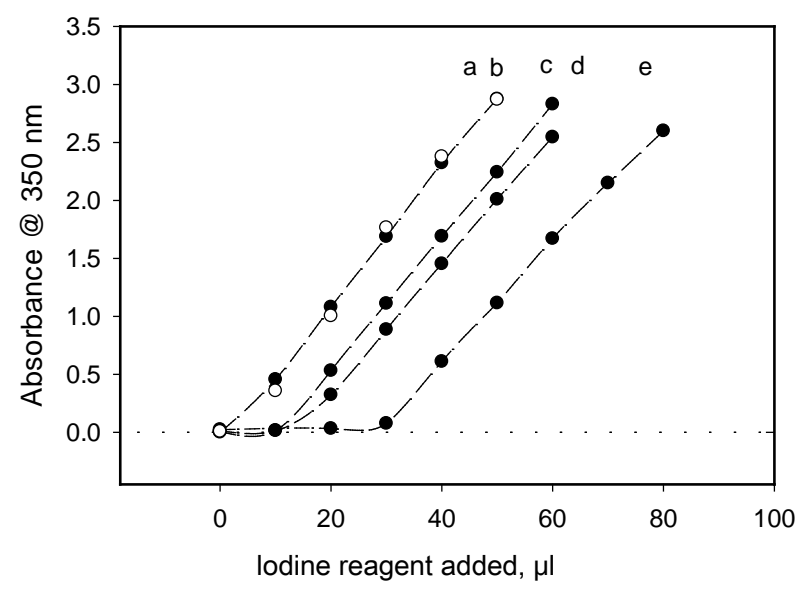

Figure 9. The iodine titration plots of various samples of amoxicillin with different expiration dates: (a) bulk buffer solution, $\mathrm{pH}$ 7.0; (b) 06/2017; (c) 03/2014; (d) 01/2013; (e) 06/2008. Solutions of amoxicillin were freshly prepared as described in M\&M. The concentration of amoxicillin in the starting solution of $20 \mathrm{mM} \mathrm{Na}-\mathrm{P}$ buffer, pH 7.0, was $2.5 \mathrm{mg} / \mathrm{mL}$. 


\section{Acknowledgements}

The research reported here was funded by the NASA Human Research Program (HRP), San Jose State University Bridges Program, and the NASA contract to the Carnegie Mellon University Silicon Valley \# NNX08AB$13 \mathrm{~A}$.

\section{References}

[1] Cielecka-Piontek, J., Michalska, K., Zalewski, P. and Jelińska, A. (2011) Recent Advances in Stability Studies of Carbapenems. Current Pharmaceutical Analysis, 7, 213-227. http://dx.doi.org/10.2174/157341211797457989

[2] El-Shaboury, S., Saleh, G., Mohamed, F. and Rageh, A. (2007) Analysis of Cephalosporin Antibiotics. Journal of Pharmaceutical and Biomedical Analysis, 45, 1-19. http://dx.doi.org/10.1016/j.jpba.2007.06.002

[3] Cielecka-Piontek, J., Michalska, K., Zalewski, P. and Zasada, S. (2012) Comparative Review of Analytical Techniques for Determination of Carbapenems. Current Analytical Chemistry, 8, 91-115. http://dx.doi.org/10.2174/157341112798472206

[4] Sawai, T., Takahashi, I. and Yamagishi, S. (1978) Iodometric Assay Method for Beta-Lactamase with Various BetaLactam Antibiotics as Substrates, in Antimicrob. Agents Chemother, 13, 910-913. http://dx.doi.org/10.1128/AAC.13.6.910

[5] Cielecka-Piontek, J., Paczkowska, M., Lewandowska, K., Barszcz, B., Zalewski, P. and Garbacki, P. (2013) SolidState Stability Study of Meropenem-Solutions Based on Spectrophotometric Analysis. Chemistry Central Journal, 7, 98. http://journal.chemistrycentral.com/content/7/1/98 http://dx.doi.org/10.1186/1752-153x-7-98

[6] Al-Abachi, M.Q., Haddi, H. and Al-Abachi, A.M. (2005) Spectrophotometric Determination of Amoxicillin by Reaction with N,N-Dimethyl-p-Phenylenediamine and Potassium Hexacyanoferrate(III). Analytica Chimica Acta, 554, 184189. http://dx.doi.org/10.1016/j.aca.2005.08.030

[7] Cielecka-Piontek, J., Zalewski, P., Krause, A. and Milewski, M. (2012) Spectrophotometric Methods as Solutions to Pharmaceutical Analysis of $\beta$-Lactam Antibiotics. In: Uddin, J., Ed., Macro To Nano Spectroscopy, InTech. http://www.intechopen.com/books/macro-to-nano-spectroscopy/spectrophotometric-methods-as-slutions-to-pharmaceu tical-analysis-of-lactam-antibiotics

[8] Salem, H. and Saleh, G. (2002) Selective Spectrophotometric Determination of Phenolic $\beta$-Lactamantibiotics. Journal of Pharmaceutical and Biomedical Analysis, 28, 1205-1213. http://dx.doi.org/10.1016/S0731-7085(02)00027-4

[9] Al-Momani, I. (2001) Spectrophotometric Determination of Selected Cephalosporins in Drug Formulations Using Flow Injection Analysis. Journal of Pharmaceutical and Biomedical Analysis, 25, 751-757. http://dx.doi.org/10.1016/S0731-7085(01)00368-5

[10] Saleh, G., El-Shaboury, S., Mahomed, F. and Rageh, A. (2009) Kinetic Spectrophotometric Determination of Certain Cephalosporins Using Oxidized Quercetin Reagent. Spectrochimica Acta, 73, 946-954. http://dx.doi.org/10.1016/j.saa.2009.05.002

[11] Ayad, M., Shalaby, A., Abdellatif, H. and Elsaid, H. (1999) Spectrophotometric Determination of Certain Cephalosporins through Oxidation with Cerium(IV) and 1-Chlorobenzotriazole. Journal of Pharmaceutical and Biomedical Analysis, 20, 557-564. http://dx.doi.org/10.1016/S0731-7085(99)00014-X

[12] Sastry, C., Rao, S., Naidu, P. and Srinivas, K. (1998) New Spectrophotometric Method for the Determination of Some Drugs with Iodine and Wool Fast Blue BL. Talanta, 45, 1227-1234. http://dx.doi.org/10.1016/S0039-9140(97)00237-3

[13] Morteza, A., Absalan, G. and Ershadifar, H. (2015) Highly Sensitive Colorimetric Determination of Amoxicillin in Pharmaceutical Formulations Based on Induced Aggregation of Gold Nanoparticles. Spectrochimica Acta Part A: Molecular and Biomolecular Spectroscopy, 143, 223-229. http://dx.doi.org/10.1016/j.saa.2015.01.071

[14] Wagman, G.H. and Weinstein, M.J. (1973) Chromatography of Antibiotics. Elsevier Scientific Pub. Co., Amsterdam.

[15] Kar, A. (2005) Pharmaceutical Drug Analysis. New Age International Publisher, New Delhi.

[16] Carstensen, J.T. and Rhodes, C. (2008) Drug Stability, Principles and Practices. In: Drugs and the Pharmaceutical Sciences, Marcel Dekker, New York, 637-750.

[17] Naidoo, K.K., Nompuku, P., Mkalali, S.N., Shabangu, K., Nkabinde, L. and Singh, V. (2006) Post-Marketing Stability Surveillance: Amoxicillin. South Africa Family Practice Journal, 48, 2078-6204.

http://dx.doi.org/10.1080/20786204.2006.10873401 